\title{
Mechanisms for the Production of Injuries in the Case of Road Traffic Accidents
}

\author{
Lucia Cerasela Balan \\ Faculty of Economic Sciences and Public Administration, Suceava, Romania,_Lucya_9212@yahoo.com
}

\begin{abstract}
The phenomenon of road traffic accidents is a global problem and is an active and growing phenomenon. The main factor contributing to road traffic accidents is the human factor. The main simple mechanisms that cause traumatic injuries in road accidents are: The impact mechanism, the most common mechanism of injuries; The fall mechanism is present when traumatic injuries are the result of contact with a rough plane such as soil or other vehicle; The design mechanism is a simple mechanism that targets both vehicle occupants and pedestrians; The limestone is the passage over the victim of the rolling stock of the vehicle; Hyperrendemia and / or hyperreflexia of the spine, this mechanism is frequently the one responsible for the determination of traumatic vertebral-medullary lesions, also called whiplash.
\end{abstract}

KEYWORDS: mechanisms, phenomenon, the blow, traffic accidents, traumatic injuries

\section{Introduction}

Human, professional and economic development and development are normal and natural processes in a world that is constantly changing.

With advancing to the better, better and prosperous, the life of the average human has improved considerably. But as every good part comes with a negative side, there has been a growing concern at the global level. Statistics are self-reported, and annual death due to a road accident is third in the world, or even second in some countries. It is ironic that besides the worrying position of this phenomenon, the statistics also reveal that the road accident phenomenon mainly targets young people from the active age group.

When talking about road traffic accidents, we are talking about a real "illness" of modern civilization, which implies more serious involvement in the prevention of road traffic accidents and their consequences (Buzatu 2009, 16).

Practice demonstrates that each traffic violation carries the phenomenon of traffic accident due to non-compliance with one or more traffic rules. Lack of road discipline is caused either by noncompliance with road rules or by ignoring them by traffic participants, who are actively involved in road traffic, which means that day-to-day must be subject to road traffic.

\section{Factors for determining road injuries}

\section{A. Main factor}

The human being; a good overall physical and mental health is an indispensable condition for the driver. Human error is the main cause of accidents, and poor health contributes to this. Thus, the obligation to assess the health status of people seeking to obtain a driving license is obvious.

Any driver must always have in mind the notion that braking is not synonymous with stopping the car. The vehicle continues its travel after braking, the distance traveled being proportional to the high square speed.

Circulation accidents are usually caused by the fact that, in the face of an abnormal unexpected event, the one behind the wheel fails to make only the automatic, ordinary but insufficient gesture. In order to avoid an accident, it is often necessary to have a great deal of spirit, in order to find a quick solution, an act that involves the contribution of the brain.

Most of the time, drivers under the influence of alcohol commit visible violations of traffic rules, such as speeding overtaking, overtaking and taking dangerous turns, non-assurance at intersections.

The intense traffic and the particular agglomeration, which characterize the road traffic nowadays, require judicious and precise regulation measures aimed at ensuring the safety of the citizens who inhabit the public roads. Ideally, for any driver, it is to be certain that the other drivers and pedestrians 
with whom they come into contact are well aware and conscientious in not complying with the obligations and provisions of the current legislation on road traffic.

\section{B. Secondary Factor}

Vehicle used in traffic; here comes the obligation to meet all the technical norms that allow technical safety on a public road - the necessity to achieve the I.T.P at 2 years.

The technical factor is generally represented by active safety, which is achieved by achieving with maximum reliability the steering, braking, lighting and signaling systems. Ensuring easy and fast maneuverability of steering systems (power steering) makes the vehicle's command response much quicker and more efficient.

Minimum start time, maximum overrun acceleration capability and maximum braking capacity are dynamic parameters that particularly affect road traffic safety, avoiding accidents.

The prevention of traffic accidents due to vehicle technical defects can be accomplished by periodically checking the machine's technical condition. Any vehicle running on public roads must comply with road safety technical regulations, environmental protection and intended use.

Although this requirement is imperative by law, however, some drivers ignore it by postponing the regular technical inspection.

\section{The circumstantial factor}

Weather, day-night discrepancy (visibility), influence of alcohol or drug substance, etc.

Road factors can be: inadequate condition of the road (holes, bumps, landslides), lack of adequate signage (curves, narrow road, slope), the existence of obstacles on the carriageway (construction materials), lack of signing of works on the road.

The road is an important factor for achieving traffic safety, increasing the fluency and comfort of road traffic. These road attributes need to be taken seriously in view of the considerable increase in the number of vehicles. Setting up and adapting roads in the increasingly intense and faster traffic is an essential condition in preventing traffic accidents (Burke 2007, 19).

\section{Mechanisms for the production of injuries in the case of road traffic accidents}

"Trauma" means any trauma suffered by the human body which, from the forensic point of view, must necessarily follow injuries caused by or within a means of transport.

From the legal point of view, the way of realization of the trauma can be revealed by the statements of the witnesses, the data of the investigation recorded in the minutes, etc.

From a forensic point of view, it can be done only by the medical examiner of the victim and / or the medical documents belonging to him / her. The identification and recording of traumatic injuries corroborated with data resulting from the corroboration of the case can objectively reveal the evidence of accused trauma.

\section{Types of mechanisms that cause traumatic injuries in road accidents}

\section{A. The blow}

The simple striking mechanism is one of the most common mechanisms for causing injuries in road accidents.

The blow can be:

- Active hitting when the object causing the trauma is in motion and hits the human body, which is in a static state;

- Passive hitting when the human body is in a dynamic state of motion and strikes a surface that may be static or dynamic; (Iftenie 2006, 93).

\section{Types of mechanisms that cause traumatic injuries in road accidents}

a) When the exterior of the vehicle moves around the human body;

b) Injuries caused by the simple knocking of one part of the interior of the vehicle in the case of a vehicle's impact on another vehicle; 
c) Crushing with a heavy body carried by means of transport, which can be fixed by the vehicle carrying it or by displacement-designing.

Generally, the severity of lesions produced by the simple hit mechanism is low. Specific lesions are characterized by bruises, excoriations and wounds, often accompanied by joint injuries and fractures. In the case of fracture injuries, areas for their production are pelvic limbs (for pedestrians) or head restraints (for occupants).

\section{B. Falling}

The fall consists in the passive hitting of the body attracted by the gravitational force of a tough body, it is encountered, almost exclusively in the case of the occupants, either when it accelerates or when it suddenly falls down.

As a result, traumatic injuries are the result of violent contact with a rough plan such as soil or other smaller vehicle stationed or in motion. The hypothetical situation of dropping a pedestrian, whether due to the blow, of the moving air column caused by the passing and high speed of a motor vehicle or because it was scared and, in desire to avoid the vehicle has been prevented and fallen, although possible, is more rarely encountered in practical work.

\section{Designing}

Designing is a simple mechanism that is common in road accidents and targets not only occupants of the vehicle but also pedestrians. Passenger design is an immediate process, while in the case of pedestrians, their design is a pre-impact effect, which in this case will be the simple trigger mechanism of the design. Passengers of a car are normally designed inside the car body. The injuries suffered by them are due to the hitting of the machine components. Typically, occupants of the rear seats are designed at the front of the head, producing traumatic brain injuries to both or outside the car.

The lesions due to the design mechanism are included in external trauma, both in the skin and internal. If external traumatic injuries are of low severity, internal ones are diverse and severe, and can be represented by fractures, visceral burglaries and hemorrhage, most of which are fatal.

\section{Compression}

Simple compression mechanism involves the crushing of the body between two harsh planes represented by: a part of the means of transport but other than the rolling system, and a rough plane, such as a wall, a tree, soil (runway), etc. or another vehicle in the case of external compressions.

By compression, external traumatic lesions, although numerically reduced, have a well-defined localization, respectively, in areas where pressure was exerted, being represented by bruises, excoriations, wounds generated by the internal parts of the car.

\section{E. The transgression}

Ironing is a simple mechanism quite often encountered in traumatic injuries caused by road accidents and signifies the passage over the victim of the car's running system.

By ironing, a body is compressed between, on the one hand, the running gear of the means of transport (wheel, track, slip sole), and on the other hand, the rolling plane, which differentiates the compression trajectory in which as we have seen, crushing of the body occurs between a part of the vehicle other than the tread and another rough plane (Beliș 1993, 719-721).

The difference between the traumatic injuries caused by ironing with the metal wheel and those produced by the rubber wheel is that, in addition to the compression between the two planes, there is also the cutting due to a shear movement, resulting in the body detachment.

\section{F. Hyperretention and / or hyperflexia of the spine}

The simple mechanisms described above cause injuries and trauma to both car and pedestrians through a specific and mandatory contact interaction with other objects. However, the simple mechanism of hyperextension or hyperflexia does not rule out interaction with other objects to cause traumatic lesions, but these lesions and traumas can arise from the simple action of the mechanism. 
This mechanism is often responsible for the determination of traumatic vertebro-medullary traumas, also called whiplash (eng.). The whiplash effect is characteristic of a whiplash movement and we mainly encounter occupants in the event of deceleration or acceleration, the impact of the vehicle on the road or stationary.

This indirect mechanism may be:

a. Monophasic, when only hyperflexia or hyperextension occurs.

b. Biphasic, when the two movements occur successively.

This simple mechanism determines traumatic lesions which, depending on the force of the movement, can result in vertebral column fractures, those caused by hyperflexia cause anterior cuneiform compression, and hyperextension affects, in addition to the posterior vertebral and spinal dislocations, with important neurological or even fatal consequences (Vieru-Socaciu 2002, 106).

\section{Conclusions}

Road accidents are today one of the major problems of medicine, by their frequency, gravity and therapeutic aspects. They affect the active age group, constituting the first cause of death, as the frequency of the young.

Part of the traumatized persons become invalids for society either as a result of the serious psychological disorders they present (post-traumatic encephalopathies) or the reduction and decrease of work capacity, requiring moral and material support for professional re-training, in less serious cases.

The forensic medical practitioner in dealing with these problems is most often required to have the patient examined by the various specialists, and sometimes to have some of them in the committees to rule in different cases.

In connection with the examination of traumatized victims in life, the forensic doctor is often in the presence of a coma case where a thorough external examination would lead to a worsening of the condition or even the loss of the patient.

The solution to the current stage of the traffic accident problem cannot be said to be complete under the forensic aspect because a critical view on the way of interpreting the casualty of traffic accidents shows a reservation in the complete and scientific exposition on the way of injuries, most of the legionary physicians summarizing the cause and cause of death.

\section{References}

Beliș, Vladimir. 1995. Treated medical law, vol. I. Bucharest: Medical Publishing House.

Burke, Michael. 2007. Forensic medical investigation of motor vehicle accidents. Boca Raton: CRC Press.

Buzatu, Nicoleta-Elena. 2009. "Investigation of road traffic accidents. Criminalistics aspects and forensic medicine". In Magazine of students and masters students of the Faculty of Juridical and Administrative Sciences. Bucharest: Pro Universitaria Publishing House.

Iftenie, Valentin. 2006. Legal medicine. Bucharest: Great Romania Foundation Publishing House.

Vieru-Socaciu, Radu. 2002. Forensic theory and practice in the context of current legislation. Cluj-Napoca Medical University: Iuliu Haţeganu Publishing House. 